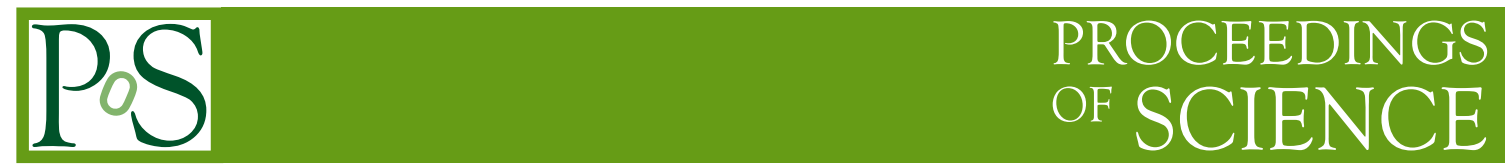

\title{
Description of moving plasma blob
}

\author{
Stanisław Ryś * \\ Astronomical Observatory, Jagiellonian University \\ strys@oa.uj.edu.pl
}

Physical models describing the structure close to the radio source's core have to account for strong forces existing in the vicinity of the black hole. Therefore, describing the motion of an outflow from the core, even in the simplest case, we should take into account a deceleration factor. Using special relativity formulae and including the orientation of the outflow direction with respect to the observer as one of the parameters, we have developed a model of an outflow close to the core. Here, a simple analytical model of decelerated motion is described and a few morphological properties of a radio source structure close to the core are discussed.

The 8th European VLBI Network Symposium

September 26-29, 2006

Toruń, Poland

${ }^{*}$ Speaker. 


\section{Introduction}

Deceleration caused by strong gravitational force exerted by supermassive black hole should be included in the description of the structures located close to a radio source's core. Thus, we should look for signatures of this effect visible in the position and brightness of the features moving away from the core, as seen in the frame of an observer located at the Earth. Throughout this paper, we term such a feature "a blob". Inclusion of the deceleration factor leads to a meaningful modification of the blob evolution deduced from a change of the blob velocity. The nature of emitting features is not well understood, but all physical models (after sophisticated calculation) provide the rules describing spectral index, position (and/or the age) of such emitting features and their brightness.

\section{Description of the blob movement}

The plasma motion is relativistic and so we have to carry out all the calculations in the framework of the special theory of relativity. Close to the active core, we can approximate the forces (i.e. deceleration) by a simple law like $\left(1 / r^{2}\right)$. We assume that deceleration vector is parallel to the velocity vector. The equation of the decelerated motion $d^{2} r / d t^{2}=c(d \beta / d t)=-g \gamma^{-3} r^{-2}$ yields after integration:

$$
\gamma=\left(\gamma_{0}-\frac{G}{r_{0}}\right)+\frac{G}{r} \quad r=\left(\gamma-\gamma_{0}\right)+\frac{G}{r_{0}}
$$

where $G=g / c^{2}, \gamma=\left(1-\beta^{2}\right)^{-1 / 2}, \beta c=d r / d t, \beta_{0}=\beta(t=0)$ and $\gamma_{0}=\left(1-\beta_{0}^{2}\right)^{-1 / 2}$, and $g$ is the amplitude of the deceleration factor. Equations (2.1) are simply the energy conservation law. We put $A=\left(\gamma_{0}-\frac{G}{r_{0}}\right)$ and so we may write (2.1) in the form: $\beta(t)=\sqrt{(A r+G)^{2}-r^{2}} /(A r+G)$. Because $\gamma_{0}>1$ implies $A>1$ and $G>0$, the solution takes the form:

$$
c\left(t-t_{0}\right)=\frac{G}{A^{2}-1}\left(\frac{A \sqrt{\gamma^{2}-1}}{\gamma-A}-\frac{1}{\sqrt{A^{2}-1}} \ln \frac{2 G}{\gamma-A}\left[\sqrt{\left(A^{2}-1\right)\left(\gamma^{2}-1\right)}+A \gamma-1\right]\right),
$$

where we can use the relation: $\gamma=(A r+G) / r$ (or $r=G /(\gamma-A))$ to calculate $t(r)$. This solution describes the time at given position (or Lorentz factor) of the moving blob.

Brightness evolution of the blob is determined by the adopted type of evolution in the rest frame of the radiating object, most frequently assumed to be (or approximated by) the power law $F(\tau) \sim v^{-\alpha} \tau^{-\mu}$, where $\alpha$ is a spectral index and $\mu$ is an index of the flux decline. The transformation rule for the time between the observer $(t)$ and the blob $(\tau)$ rest frames is: $d \tau=d t / \gamma(t)$ and using the known $\gamma(r)$ dependence we get: $c d \tau=r \cdot d r / \sqrt{(A r+G)^{2}-r^{2}}$, which leads us to an integral similar to (2.2):

$$
c\left(\tau-\tau_{0}\right)=\frac{G}{\left(A^{2}-1\right)}\left(\frac{\sqrt{\gamma^{2}-1}}{(\gamma-A)}-\frac{A}{\sqrt{A^{2}-1}} \ln \frac{2 G}{\gamma-A}\left|\sqrt{\left(A^{2}-1\right)\left(\gamma^{2}-1\right)}+A \gamma-1\right|\right) .
$$


Assuming the values of the parameters $\gamma_{0}, g, \cos (\theta)$, we are able to calculate $t$ and $r$ - the position at which we expect to find the blob. We assume that for $\tau\left(t_{o b s}=0\right)=0$ the plasma element has $\gamma=\gamma_{0}$. In the observer's rest frame, we should take into account the signal travel-time corrections introduced by the geometry in the problem under consideration. The equation relating the position and the age of the blob is: $t-(r / c) \cos (\theta)=t_{o b s}$, where $\theta$ is an angle between the line of sight and the outfbw direction. From these equations, knowing $r$ and using equation (2.2), we can calculate $t_{o b s}$. If the radiation is isotropic in the rest frame of the blob then the flux of photons received by the observer is often approximated with: $f\left(r\left(t_{o b s}\right)\right)=f_{0} \cdot D^{3+\alpha} \cdot \tau^{-\mu}$, where $\alpha$ is a spectral index and $\mu$ is an index of the flux decline. At any given distance from the core $(r)$, we can calculate $\gamma, \tau$-intrinsic age, and $t_{o b s}$ - the age of the blob in the rest frame of the observer and finally the flux $f\left(t_{o b s}\right) ; r\left(t_{o b s}\right)$ and $f\left(t_{o b s}\right)$ are determined by the values of $\cos \theta, \alpha, \mu$, and $\gamma_{0}$.

\section{Example}

In this example, we use the formula describing an evolution of the flux $f\left(t_{o b s}\right)$ of a plasma blob in the observer's rest frame (Fig. 1). For all curves presented here, it is assumed that the blob has the same value of the flux at the beginning $f(\tau=0), \gamma_{0}=4.0$ and $\alpha=0.75$. The curves in the left panel of Fig.1 represent different values of parameter $\cos (\theta)=0.0-1.0$ (with $\mu=0.2$ ), and in the right panel the curves are plotted for different values of $\mu=0.1-1.1$ (with $\cos (\theta)=0.5$ ). In the model presented here, the deceleration decreases the value of Lorentz factor $\gamma_{0}$ not more than to $\gamma_{0}-1$. If the jet starts with a high value of $\gamma_{0}$, the value of $\beta$ will be close to 1 over most of the travelled distance and we do not expect that observed velocity of the blob will change substantially. However, the change of the radio flux received by the observer could help us to detect the evolution of the blob.

We realize that the shortest jets may be very small and short living and we will never see their spatial structure but we can notice the temporal change of the flux of the whole source. The prediction of a varying flux could be compared with observational data and tested if the variation is determined by the blob escaping the vicinity of the black hole.

\section{Closing remarks}

Approaching the black hole horizon, we try to resolve the spatial structure of the AGN the region forming a jet. The VLBI maps (e.g. Kirchbaum et al. 2004) allow us to check the evolution of a single feature moving away from the core, i.e. its distance and flux. Testing any model describing this phenomenon one should discuss the forces influencing trajectories of the moving plasma and their flux evolution as well as the position-flux correlation. Inclusion of the deceleration into the standard model of the energetic outfbw from AGN is indispensable when we look at the region where the jet is forming.

\section{References}

[1] T.P. Kirchbaum, D.A. Graham, W. Alef, et al., Towards the Event Horizon - The Vicinity of AGN at Micro-Arcsecond Resolution in proceedings of 7th European VLBI Network Symposium, ed. by R. Bachiller, F. Colomer, J.F. Desmurs, P. de Vicente. 

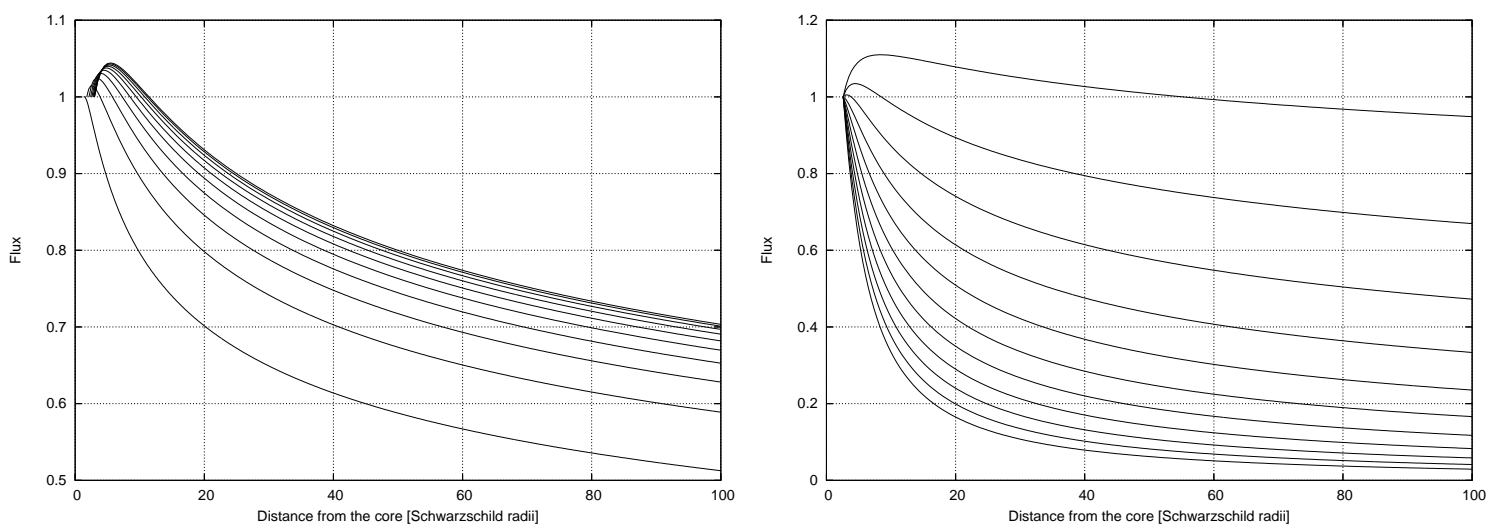

Figure 1: Theoretical dependencies of the flux along the distance from the core for a few values of the model parameters: viewing angle $\cos \theta=0.0-1.0$ (left), and index of the flux decline $\mu=0-1$ (right). 